\title{
Research Article \\ Effect of a Bonded Patch on Aeroelastic Behavior of Cantilevered Plates
}

\author{
Sirwan Farhadi and Shahrokh Hosseini-Hashemi \\ School of Mechanical Engineering, Iran University of Science and Technology, Narmak, \\ Tehran 16846-13114, Iran \\ Correspondence should be addressed to Sirwan Farhadi, sirwan@iust.ac.ir
}

Received 7 April 2010; Accepted 17 June 2010

Academic Editor: George Jaiani

Copyright (C) 2010 S. Farhadi and S. Hosseini-Hashemi. This is an open access article distributed under the Creative Commons Attribution License, which permits unrestricted use, distribution, and reproduction in any medium, provided the original work is properly cited.

\begin{abstract}
In recent years, many researchers have studied vibration suppression of fluttering plates using piezoelectric actuators. Lots of these researchers have focused on optimal placement of piezoelectric patches to obtain maximum controllability. Although mass and stiffness characteristics of bonded patches can alter aeroelastic behavior of fluttering plates, few of them considered the effect of the mentioned parameters in optimization process. This paper investigates effect of a bonded patch on aeroelastic behavior of cantilevered plates in supersonic flow. For this purpose, critical dynamic pressure and limit-cycle oscillations of the system are studied. Von Karman plate theory along with first order piston theory is employed for mathematical simulation of the system. Obtained results reveal that a bonded patch with a small mass ratio can change the system critical dynamic pressure significantly, where the main part of the variations is resulted from the added mass of the bonded patch. The maximum raise of dynamic pressure is acquired when the patch is placed on the plate's leading edge. The results show that mass and stiffness characteristics of bonded piezoelectric patches can have a great impact on aeroelastic performance of fluttering plates. Therefore, these parameters must be considered as effective factors for optimal placement of piezo-actuators.
\end{abstract}

\section{Introduction}

Recently, performance optimization of smart materials and structures has attracted many researchers. Lots of them focused on the optimal vibration suppression of fluttering plates and panels which are ideal models for wings and membrane elements of airplanes and missiles. For this aim, one of challenges was to obtain optimal location of piezoelectric actuators. Therefore lots of works were devoted to optimal placement of the bonded actuators. Most of researchers used controllability-based optimization methods for maximizing system controllability index (e.g., see [1-4]). Although, in specific patch positions, mass and stiffness 
characteristics of the bonded patches can considerably alter aeroelastic behavior of fluttering plates, few researchers considered variations of system dynamics in the optimization process.

Nam et al. [5] accounted for patch mass and stiffness effect in their optimization method by including closed-loop damping ratios as design constraints in optimization objective function. Richard and Clark [6] included patch mass and stiffness effects within their optimization routine indirectly by introducing a metric which presents a rough prediction of the effects of patch mass and stiffness contributions on the system.

According to our survey, no study is published in the literature focusing on the effect of patch position on dynamic response of plates subjected to air flow. In the present paper, our special attention is to study effect of bonded patches on critical dynamic pressure of cantilevered plates and vibration amplitude of fluttering plates.

Von Karman plate theory [7] is employed for mathematical simulation of the structure which reduces computational efforts considerably in comparison with higher-order plate theories. A recent study by Amabili and Farhadi [8] shows that, for isotropic and multilayer composite plates, nonlinear vibration behavior predicted by Von Karman plate theory is almost identical with that predicted by higher-order plate theories if the computed natural frequencies from these theories are the same. It is worth mentioning that natural frequencies calculated by Von Karman theory are in a good agreement with those of higher-order plate theories if the lateral shear deformations are negligible. Since we use a thin cantilevered plate and only a single patch with a small thickness ratio is bonded to the plate, this case is true for our survey. In this study, embedded piezoelectric actuator is simply modeled as conventional structural materials and its piezoelectric properties are ignored.

Air flow pressure is modeled using first-order plate theory. This aerodynamic theory is valid for irrotational nonviscous air flows and limits our survey to supersonic region. Also, Kane and Levinson's dynamic method [9] is used to obtain nonlinear governing equations of motion. Critical dynamic pressure of the structure is determined ignoring nonlinear terms in the governing equations. In order to obtain maximum critical dynamic pressure, the patch is placed in different positions and critical dynamic pressure is calculated. Then, effect of bonded patch on limit-cycle oscillations of the fluttering plate is investigated. The obtained results show that bonding a patch with a small mass ratio to a cantilevered plate can effectively alter its critical dynamic pressure and amplitude of limit-cycle oscillations. In fact, by placing a patch on the plate, vibration mode shapes of the plate change. Hence, aerodynamic couplings between vibrating modes are magnified or restrained.

Current methods of patch placement are based on system controllability optimization. In these methods, it is assumed that effect of patch's structural characteristics on system dynamic is negligible and simply system controllability index is investigated. However this statement is not valid for cantilevered plates subjected to lateral supersonic flows.

While the main aim of investigators is to suppress vibrations of fluttering plates, presented results suggest utilization of hybrid method for vibration stabilization of the mentioned systems. These results suggest that there is a possibility to find actuator locations where the controllability index is not optimal; instead the aeroelastic characteristics of the system are modified so that the needed actuator effort reduces and therefore the overall control performance improves considerably.

\section{Governing Equations}

Figure 1 shows a cantilevered plate with length $a$ and thickness $b$ subjected to a lateral supersonic flow of velocity $V_{\infty}$. This plate is embedded with a piezoelectric patch of 


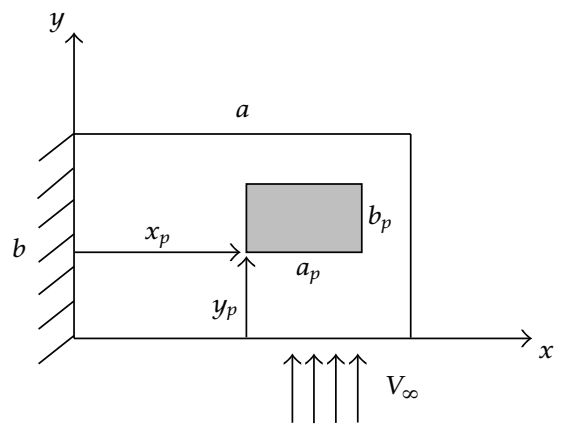

(a)

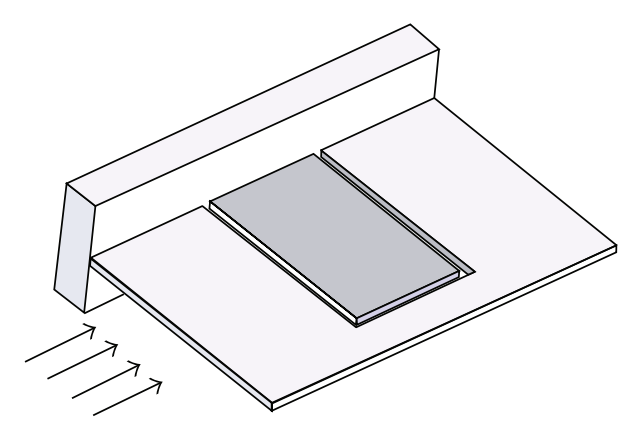

(b)

Figure 1: (a) A cantilevered plate with a bonded patch and subjected to a lateral supersonic flow as well as (b) a baffled plate.

dimensions $a_{P}$ and $b_{P}$ located in coordinates $\left(x_{P}, y_{P}\right)$. To guarantee the uniformity of the lateral flow, a baffled plate is considered (Figure 1(b)).

According to the Kane dynamic method [9], governing equations of motion are expressed as:

$$
\mathbf{F}^{*}+\mathbf{F}-\mathbf{F}_{e x}=\mathbf{0},
$$

where $\mathbf{F}^{*}, \mathbf{F}$, and $\mathbf{F}_{e x}$ are vectors of the generalized inertia forces, the generalized internal active forces, and the generalized external active forces, respectively, which are defined as follows:

$$
\begin{gathered}
\mathbf{F}^{*}=\int_{V} \rho \frac{\partial \mathbf{v}_{\mathbf{P}}}{\partial \dot{\mathbf{q}}} \cdot \mathbf{a}_{\mathbf{P}} d V, \\
\mathbf{F}=\frac{\partial U}{\partial \mathbf{q}} \\
\mathbf{F}_{e x}=\int_{V} \frac{\partial \dot{\mathrm{w}}}{\partial \dot{\mathbf{q}}} \mathrm{P} d A,
\end{gathered}
$$

where $\dot{\mathbf{q}}$ stands for time derivative of the generalized coordinates vector $\mathbf{q}$ while $\mathbf{v}_{\mathbf{P}}$ and $\mathbf{a}_{\mathbf{P}}$ are denoted for velocity and acceleration vectors of a generic point $p$, respectively, and $\dot{w}$ is lateral velocity of the plate. In addition, $P$ is the aerodynamic pressure and $U$ is the strain energy of the structure.

Velocity and acceleration vectors can be obtained as follows:

$$
\mathbf{v}_{\mathbf{P}}=\dot{u}_{0} e_{1}+\dot{v}_{0} e_{2}+\dot{w}_{0} e_{3}, \quad \mathbf{a}_{\mathbf{P}}=\ddot{u}_{0} e_{1}+\ddot{v}_{0} e_{2}+\ddot{w}_{0} e_{3},
$$

where $u_{0}, v_{0}$, and $w_{0}$ denote the midplane deformations along the $x_{-}, y^{-}$, and $z$-axes, respectively, and $e_{1}, e_{2}$, and $e_{3}$ stand for the corresponding unite vectors. 
Strain energy of the structure is calculated by

$$
U=\frac{1}{2} \int_{V} \varepsilon^{\mathrm{T}} \mathbf{C} \varepsilon d V
$$

where $\mathbf{C}$ is the material stiffness matrix, $\varepsilon$ is vector of strain components, and $V$ is total volume of the structure.

According to Von Karman plate theory, components of strain field are obtained through the following equation:

$$
\begin{gathered}
\varepsilon_{x x}=\frac{\partial u_{0}}{\partial x}+\frac{1}{2}\left(\frac{\partial w_{0}}{\partial x}\right)^{2}-z \frac{\partial^{2} w_{0}}{\partial x^{2}} \\
\varepsilon_{y y}=\frac{\partial v_{0}}{\partial y}+\frac{1}{2}\left(\frac{\partial w_{0}}{\partial y}\right)^{2}-z \frac{\partial^{2} w_{0}}{\partial y^{2}} \\
\varepsilon_{x y}=\frac{\partial u_{0}}{\partial y}+\frac{\partial v_{0}}{\partial x}+\left(\frac{\partial w_{0}}{\partial x} \frac{\partial w_{0}}{\partial y}\right)-2 z \frac{\partial^{2} w_{0}}{\partial x \partial y}
\end{gathered}
$$

and strain vector is formed as below:

$$
\boldsymbol{\varepsilon}=\left[\begin{array}{lll}
\varepsilon_{x x} & \varepsilon_{y y} & \varepsilon_{x y}
\end{array}\right]^{T} .
$$

First-order piston theory is employed to obtain the aerodynamic pressure. This theory is used for steady flows with Mach number higher than 1.6 and determines air dynamic pressure as follows [11]:

$$
P=-\frac{\rho_{a} \mathrm{~V}_{\infty}^{2}}{\beta}\left(\frac{\partial w}{\partial y}+\frac{1}{\mathrm{~V}_{\infty}}\left(\frac{M_{\infty}^{2}-2}{M_{\infty}^{2}-1}\right) \frac{\partial w}{\partial t}\right)
$$

In the above equation, $\rho_{a}$ and $M_{\infty}$-denote the air density and free-stream Mach number, respectively, and $\beta$ is a dimensionless number defined as $\beta=\sqrt{M_{\infty}^{2}-1}$.

Using Ritz method, displacement field components can be approximated as follows:

$$
\begin{aligned}
& u_{0}=\sum_{j=1}^{N_{1}} \sum_{i=1}^{M_{1}} a_{i+(j-1) M_{1}} G_{1}(x, y) \Phi_{i}(x) \Psi_{j}(y), \\
& v_{0}=\sum_{j=1}^{N_{2}} \sum_{i=1}^{M_{2}} b_{i+(j-1) M_{2}} G_{2}(x, y) \Phi_{i}(x) \Psi_{j}(y), \\
& w_{0}=\sum_{j=1}^{N_{3}} \sum_{i=1}^{M_{3}} c_{i+(j-1) M_{3}} G_{3}(x, y) \Phi_{i}(x) \Psi_{j}(y),
\end{aligned}
$$

where $a_{i+(j-1) M_{1}}, b_{i+(j-1) M_{2}}$, and $c_{i+(j-1) M_{3}}$ are time-dependent coefficients, $\Phi_{i}(x)$ and $\Psi_{j}(y)$ are arbitrary functions, $G_{i}(x, y)$ are manipulator functions satisfying geometrical boundary 
conditions, and $N_{1}, N_{2}, N_{3}$ and $M_{1}, M_{2}, M_{3}$ are the numbers of terms used in the approximation functions in $x$ and $y$ directions, respectively. For a plate cantilevered in the edge $x=0$, the manipulator functions are obtained as $G_{i}(x, y)=x, i=1,2,3$. For convenience, the above-assumed displacement functions are expressed in the matrix form as:

$$
\mathbf{d}=\left\{\begin{array}{c}
u_{0} \\
v_{0} \\
w_{0}
\end{array}\right\}=\mathbf{N q}, \quad \mathbf{q}=\left\{\begin{array}{l}
\mathbf{a} \\
\mathbf{b} \\
\mathbf{c}
\end{array}\right\}
$$

where $\mathbf{N}$ is the matrix of shape functions, $\mathbf{q}$ is the generalized coordinates vector, and $\mathbf{a}, \mathbf{b}$, and $\mathbf{c}$ are vectors made of the corresponding time-dependent coefficients reported in (2.8).

Introducing the following operator matrices:

$$
\begin{gathered}
\mathbf{H}_{\mathbf{1}}=\left[\begin{array}{lll}
1 & 0 & 0
\end{array}\right], \quad \mathbf{H}_{\mathbf{2}}=\left[\begin{array}{lll}
0 & 1 & 0
\end{array}\right], \\
\mathbf{H}_{3}=\left[\begin{array}{llll}
0 & 0 & 1
\end{array}\right], \quad \mathrm{H}_{4}=\left[\begin{array}{lll}
\frac{\partial}{\partial y} & 0 & 0
\end{array}\right], \\
\mathbf{L}_{\mathbf{i}}=\mathbf{H}_{\mathbf{i}} \mathbf{N}, \quad \mathbf{Q}_{\mathbf{i}}=\mathbf{A}_{\mathbf{i}} \mathbf{N}, \quad \mathbf{S}_{\mathbf{j}}=\mathbf{B}_{\mathbf{j}} \mathbf{N}
\end{gathered}
$$

and substituting (2.3), (2.4), and (2.7) into (2.2), the generalized equations of motion are derived as follows:

$$
\mathbf{M} \ddot{\mathbf{q}}+\mathbf{C}_{\mathbf{A}} \dot{\mathbf{q}}+\left(\mathbf{K}+\mathbf{K}_{\mathbf{q}}+\mathbf{K}_{\mathbf{q q}}+\mathbf{K}_{\mathbf{A}}\right) \mathbf{q}=\mathbf{0}
$$

where $\mathbf{M}, \mathbf{C}_{\mathbf{A}}$, and $\mathbf{K}_{\mathbf{A}}$ are denoted as mass matrix, aerodynamic damping matrix, and aerodynamic stiffness matrix, respectively, and $\mathbf{K}, \mathbf{K}_{\mathbf{q}}$, and $\mathbf{K}_{\mathbf{q q}}$ stand for linear, quadratic, and cubic stiffness matrices, in that order. These matrices are introduced as given below:

$$
\begin{gathered}
\mathbf{M}=\rho \int_{V}\left(\mathbf{L}_{1}{ }^{\mathrm{T}} \mathbf{L}_{1}+\mathbf{L}_{2}{ }^{\mathrm{T}} \mathbf{L}_{2}+\mathbf{L}_{3}{ }^{\mathrm{T}} \mathbf{L}_{3}\right) d V, \\
\mathbf{K}=\int_{V} \mathbf{S}_{1}{ }^{\mathrm{T}} \mathbf{C} \mathbf{S}_{1} d V, \\
\mathbf{K}_{\mathbf{q}}=\int_{V}\left(\mathbf{S}_{1}{ }^{\mathrm{T}} \mathbf{C D}_{1}{ }^{\mathrm{T}}+\left(\mathbf{D}_{1}+\mathbf{D}_{2}\right) \mathbf{C} \mathbf{S}_{1}\right) d V, \\
\mathbf{K}_{\mathbf{q q}}=\int_{V}\left(\mathbf{D}_{1}+\mathbf{D}_{2}\right) \mathbf{C D}_{1}{ }^{\mathrm{T}} d V,
\end{gathered}
$$




$$
\begin{gathered}
\mathbf{C}_{\mathbf{A}}=\frac{\rho_{a} \mathrm{~V}_{\infty}}{\beta}\left(\frac{M_{\infty}^{2}-2}{M_{\infty}^{2}-1}\right) \int_{A} \mathbf{L}_{3}{ }^{\mathrm{T}} \mathbf{L}_{3} d A, \\
\mathbf{K}_{\mathbf{A}}=\frac{\rho_{a} \mathrm{~V}_{\infty}^{2}}{\beta} \int_{A} \mathbf{L}_{3}{ }^{\mathrm{T}} \mathbf{L}_{4} d A .
\end{gathered}
$$

In (2.12)-(2.17), the following stiffness and operator matrices are used:

$$
\begin{aligned}
& \mathbf{C}=\left[\begin{array}{ccc}
C_{11} & C_{12} & 0 \\
C_{12} & C_{22} & 0 \\
0 & 0 & C_{66}
\end{array}\right] \\
& \mathbf{B}_{1}=\left[\begin{array}{ccc}
\frac{\partial}{\partial x} & 0 & -z \frac{\partial^{2}}{\partial x^{2}} \\
0 & \frac{\partial}{\partial y} & -z \frac{\partial^{2}}{\partial y^{2}} \\
\frac{\partial}{\partial y} & \frac{\partial}{\partial x} & -2 z \frac{\partial^{2}}{\partial x \partial y}
\end{array}\right], \quad \mathbf{B}_{2}=\left[\begin{array}{lll}
0 & 0 & \frac{\partial}{\partial x} \\
0 & 0 & \frac{\partial}{\partial y} \\
0 & 0 & \frac{\partial}{\partial y}
\end{array}\right], \\
& \mathbf{A}_{1}=\left[\begin{array}{ccc}
0 & 0 & \frac{1}{2} \frac{\partial}{\partial x} \\
0 & 0 & 0 \\
0 & 0 & 0
\end{array}\right], \quad \mathbf{A}_{2}=\left[\begin{array}{ccc}
0 & 0 & 0 \\
0 & 0 & \frac{1}{2} \frac{\partial}{\partial y} \\
0 & 0 & 0
\end{array}\right], \quad \mathbf{A}_{3}=\left[\begin{array}{ccc}
0 & 0 & 0 \\
0 & 0 & 0 \\
0 & 0 & \frac{\partial}{\partial x}
\end{array}\right], \\
& D_{1}=\left\lfloor\begin{array}{llll}
S_{2}{ }^{\mathrm{T}} \mathbf{Q}_{1} \mathbf{q} & \mathrm{S}_{2}{ }^{\mathrm{T}} \mathbf{Q}_{2} \mathbf{q} & \mathrm{S}_{2}{ }^{\mathrm{T}} \mathbf{Q}_{3} \mathbf{q}
\end{array}\right], \\
& \mathbf{D}_{2}=\left[\begin{array}{lllll}
\mathbf{Q}_{1}{ }^{\mathrm{T}} \mathbf{S}_{2} \mathbf{q} & \mathbf{Q}_{2}{ }^{\mathrm{T}} \mathbf{S}_{2} \mathbf{q} & \cdots & \mathbf{Q}_{3}{ }^{\mathrm{T}} \mathbf{S}_{2} \mathbf{q}
\end{array}\right] .
\end{aligned}
$$

In (2.12)-(2.15), the introduced integrals are calculated over total volume including volume of the plate and the patch.

\section{Critical Dynamic Pressure}

Omitting nonlinear terms from (2.11), the following linear equation is obtained:

$$
\mathbf{M} \ddot{\mathbf{q}}+\mathbf{C}_{\mathbf{A}} \dot{\mathbf{q}}+\left(\mathbf{K}+\mathbf{K}_{\mathbf{A}}\right) \mathbf{q}=\mathbf{0} .
$$


Assuming the generalized coordinates vector to be harmonic $\left(\mathbf{q}=\overline{\mathbf{q}} e^{i \omega t}\right)$, this equation reduces to a conventional eigenvalue problem:

$$
\left(\mathbf{M} \alpha^{2}-\mathbf{C}_{\mathbf{A}} \alpha+\mathbf{K}+\mathbf{K}_{\mathrm{A}}\right) \overline{\mathbf{q}}=\mathbf{0},
$$

where the corresponding eigenvalues depend on flow characteristics and in general form are complex values, as follows:

$$
\alpha=\eta+i \omega
$$

In the above equation, $\omega$ specifies vibration harmony and $\eta$ shows damping rate.

In aeroelasticity field, the system behavior is commonly discussed in terms of dimensionless dynamic pressure $\lambda$, which is given as

$$
\lambda=\frac{\rho_{a} V_{\infty}^{2} a^{3}}{D}, \quad D=\frac{C_{11} h^{3}}{12}
$$

where $D$ and $h$ stand for flexural rigidity of the plate and plate thickness, respectively, and $C_{11}$ indicates the first element of the plate stiffness matrix.

Solving characteristic equation (3.2) for different values of dimensionless dynamic pressures, it is observed that, for small values of $\lambda$, plate damping rate $\eta$ is negative, which indicates that the plate vibrations decay gradually and system is stable. On the other hand, damping rate value $\mu$ becomes positive and plate oscillations turn into unstable zone when dynamic pressure goes beyond a critical value which is introduced by $\lambda_{c r}$. In practice, existence of nonlinear stiffness terms in the governing equation limits the amplitude of vibration to a bounded value.

\section{Numerical Results}

In the present paper, power series are used as arbitrary functions $\left(\Phi_{i}(x)=x^{i-1}\right.$ and $\Psi_{j}(y)=$ $\left.y^{j-1}\right)$. For convenience, the approximation series in different directions are taken of the same order $\left(M_{1}=M_{2}=M_{3}=M\right.$ and $\left.N_{1}=N_{2}=N_{3}=N\right)$. Presented results are obtained in terms of the following dimensionless values:

$$
\begin{gathered}
\eta=\frac{a}{b}, \quad \delta=\frac{h}{a}, \quad \bar{a}=\frac{a_{p}}{a}, \\
\bar{b}=\frac{b_{p}}{a}, \quad \bar{h}=\frac{h_{p}}{h}, \quad X=\frac{x_{P}}{a}, \\
Y=\frac{y_{P}}{b}, \quad \mu=\frac{b \rho_{a}}{h \rho} .
\end{gathered}
$$

Here, the characteristic matrices $\mathbf{M}, \mathbf{C}_{\mathbf{A}}, \mathbf{K}_{\mathbf{A}}, \mathbf{K}, \mathbf{K}_{\mathbf{q}}$, and $\mathbf{K}_{\mathbf{q q}}$ are calculated through exact analytical integrations using Mathematica software.

Table 1 presents a convergence study for critical dynamic pressure of a simply supported square plate and compares the calculated value with the exact solution of the 
Table 1: Critical dynamic pressure predicted by the current analysis for different order of approximation series and comparison with the exact solution [10].

\begin{tabular}{cccccccc}
\hline \multicolumn{7}{c}{$\mathrm{N} \times \mathrm{M}$} \\
\hline & $4 \times 4$ & $5 \times 5$ & $6 \times 6$ & $7 \times 7$ & $8 \times 8$ & Exact [10] & Error percent \\
$\lambda_{c r} / \beta$ & 627.9 & 439.7 & 520.8 & 517.6 & 518.0 & 512.6 & 1.05 \\
\hline
\end{tabular}

Table 2: Material properties of the base plate and the bonded patch.

\begin{tabular}{lccccc}
\hline & $C_{11}(\mathrm{GPa})$ & $C_{12}(\mathrm{GPa})$ & $C_{22}(\mathrm{GPa})$ & $C_{66}(\mathrm{GPa})$ & $\rho\left(\mathrm{kg} / \mathrm{m}^{3}\right)$ \\
\hline Aluminum & 76.92 & 23.08 & 76.92 & 26.92 & 2700 \\
PZT5H [12] & 130.6 & 85.66 & 135.8 & 22.99 & 7740 \\
\hline
\end{tabular}

problem [10]. This table shows that, with polynomial approximation series of order $N \times M=$ $8 \times 8$, the calculated critical dynamic pressure converges to the exact solution with an adequate precision.

In order to study patch placement effect on critical dynamic pressure, a cantilevered plate with the following dimensional values is considered:

$$
a=0.4 \mathrm{~m}, \quad \delta=\frac{4}{3}, \quad \eta=0.01 .
$$

A rectangular patch with the following dimensions is bonded to the plate:

$$
\bar{a}=\frac{1}{4}, \quad \bar{b}=\frac{5}{30}, \quad \bar{h}=\frac{1}{4} .
$$

In this paper, the free-stream Mach number is set to $M_{\infty}=2$ and air-panel mass ratio is considered as $\mu=0.1$.

An aluminum base plate is considered ( $E=70 \mathrm{GPa}$ and $v=0.3)$ and two materials are considered for the embedded patch, namely, aluminum and PZT5H. The employed material coefficients are reported in Table 2 [12].

Bonding a patch to the cantilever plate modifies the mass and stiffness matrices by some values, depending on the patch position, which in turn changes the critical dynamic pressure. Although mode shapes of the base plate are symmetric with respect to plate central axis, effect of patch placement on critical dynamic pressure is not symmetric. This asymmetry is caused by fluid flow which is unidirectional and asymmetric. Figures $2(a)$ and 2(b) exhibit variations of critical dynamic pressure versus patch position for a cantilevered plate with an aluminum patch and a cantilevered plate with a piezoelectric patch, respectively. According to the patch dimensions (4.3) and patch density (Tables 2 and 3), patch-to-plate mass ratio is .01 for the aluminum patch and is 0.03 for the piezoelectric patch. However, from Figure 2(a), it is observed that placement of these patches can increase the critical dynamic pressure of the base plate extensively. Calculations show that, for the case with the aluminum patch, maximum value of critical dynamic pressure is about $32 \%$ larger than the base value, and for the case with piezoelectric patch this value is about $50 \%$ larger than the base one. It means that bounding a patch with rather small mass ratio to a cantilevered plate alters its dynamic characteristics significantly. Figure 2(b) shows that maximum increment of critical dynamic 
Table 3: Maximum critical dynamic pressure versus dimensional ratios of the bonded patch.

\begin{tabular}{lcccccccccc}
\hline & \multicolumn{10}{c}{ Patch width ratio $\bar{b}$} \\
\hline Patch length ratio $\bar{a}$ & 0.1 & 0.2 & 0.3 & 0.4 & 0.5 & 0.6 & 0.7 & 0.8 & 0.9 & 1 \\
\hline 0.1 & 168.1 & 182.3 & 187.2 & 185.2 & 179.1 & 170.6 & 161.3 & 151.8 & 142.3 & 139.9 \\
0.2 & 188.4 & 212.9 & 219.3 & 214.5 & 203.7 & 190.0 & 175.5 & 161.1 & 147.2 & 139.5 \\
0.3 & 204.4 & 235.2 & 242.0 & 235.5 & 221.9 & 204.9 & 187.2 & 169.8 & 153.0 & 139.8 \\
0.4 & 215.7 & 250.2 & 257.2 & 249.8 & 234.7 & 215.9 & 196.2 & 176.8 & 158.0 & 140.1 \\
0.5 & 222.7 & 259.1 & 266.3 & 258.8 & 243.8 & 223.1 & 202.2 & 181.6 & 161.6 & 141.3 \\
0.6 & 226.4 & 263.6 & 271.0 & 263.4 & 247.3 & 227.0 & 205.5 & 184.3 & 163.7 & 142.5 \\
0.7 & 227.9 & 265.4 & 272.9 & 265.3 & 249.2 & 228.8 & 207.0 & 185.5 & 164.5 & 143.0 \\
0.8 & 228.3 & 265.9 & 273.4 & 265.9 & 249.7 & 229.2 & 207.3 & 185.8 & 164.7 & 143.0 \\
0.9 & 228.4 & 265.9 & 273.4 & 265.9 & 249.8 & 229.3 & 207.4 & 185.8 & 164.8 & 143.0 \\
1 & 228.4 & 265.9 & 273.4 & 265.9 & 249.8 & 229.3 & 207.4 & 185.8 & 164.8 & 143.0 \\
\hline
\end{tabular}

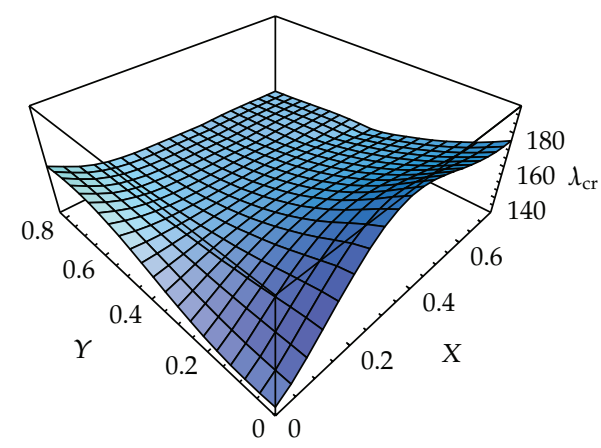

(a)

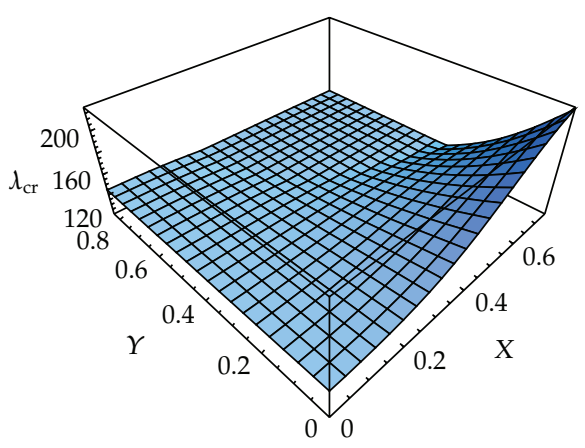

(b)

Figure 2: Variations of critical dynamic pressure versus patch location: (a) with an aluminum patch and (b) with a PZT5H patch.

pressure is achieved when the patch is bonded at the free corner on the leading edge (i.e., $(X, Y)=(0.75,0))$.

In order to assess the effects of patch density and stiffness on critical dynamic pressure, separately, two piezoelectric patches with unreal material properties are considered: one with reduced mass (90\% less than original value) and the other one with reduced stiffness $(90 \%$ less than original value). Figures $3(\mathrm{a})$ and $3(\mathrm{~b})$ demonstrate variations of critical dynamic pressure versus patch position for the patch with reduced mass and the patch with reduced stiffness, respectively. From these figures, it can easily be concluded that patch density affects critical dynamic pressure more evidently in comparison with its stiffness.

In order to optimize the size of piezoelectric patch for passive stabilization of the flow-induced vibrations, variations of critical dynamic pressure versus patch size have been investigated. Obtained results show that, for different patch sizes and aspect ratios, the optimal location for maximum critical dynamic pressure matches with the free corner of the plate on the leading edge. In addition, variations of critical dynamic pressure versus patch location follow the same pattern as presented in Figure 2(b). Maximum critical dynamic pressure versus patch dimensional ratios is presented in Table 3. From this table it can be observed that, by setting the patch length ratio $(\bar{a})$ to a fixed value and by increasing the patch 


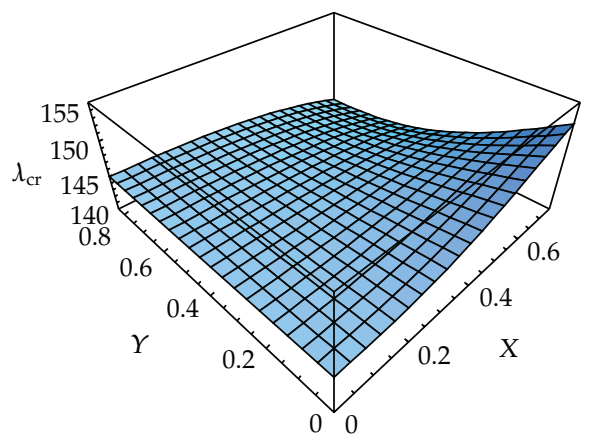

(a)

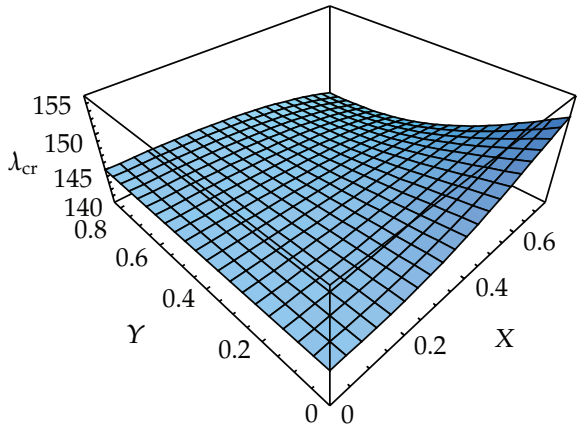

(b)

Figure 3: Variations of critical dynamic pressure versus patch location: (a) with reduced mass and (b) with reduced stiffness.

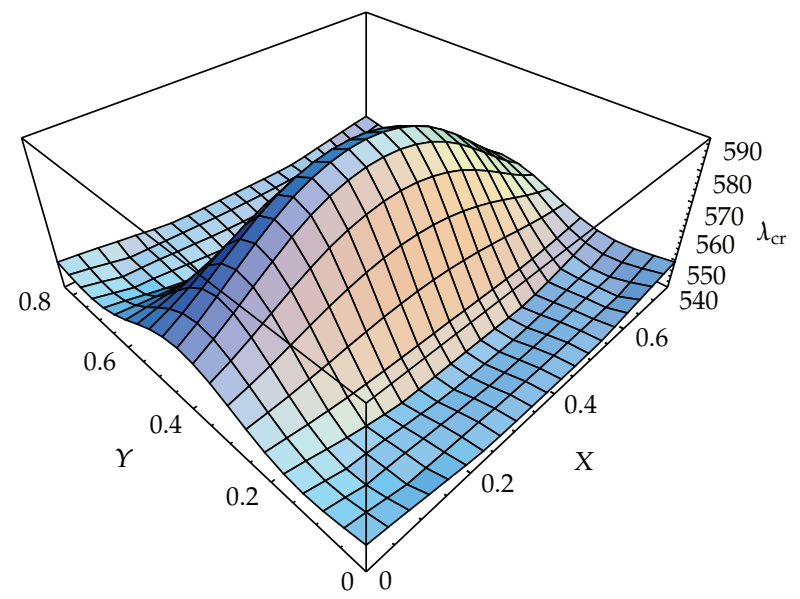

Figure 4: Variations of critical dynamic pressure versus patch location for a simply supported plate with dimensions $a=0.4 \mathrm{~m}, \delta=4 / 5$.

width ratio $(\bar{b})$ monotonically, the maximum value of critical dynamic pressure increases firstly and then decreases significantly. On the other hand, by fixing the patch width ratio $(\bar{b})$, the maximum value of critical dynamic pressure increases as the patch length ratio $(\bar{a})$ increases and converges to a boundary value.

In the sequel, a simply supported plate with dimensions $a=0.4 \mathrm{~m}, \delta=4 / 5$, and $\eta=0.01$ is considered. A piezoelectric patch with dimensional ratios presented in (4.3) is bonded to the plate. For this case, variations of critical dynamic pressure versus patch location are demonstrated in Figure 4. From this figure, it is observed that, for simply supported boundary conditions, placement of a piezoelectric patch on the plate will increase critical dynamic pressure by $10 \%$, at maximum. This reveals that aeroelastic behavior of simply supported plates is less sensitive to the embedded patches. Therefore, effect of patch placement on system dynamic can be omitted and one can use controllability-based optimization methods to obtain optimal patch location. 
Table 4: Dimensionless amplitude of limit-cycle oscillations $(\mathrm{w} / \mathrm{h})$ of a cantilevered square plate in dimensionless coordinates $(x / a, y / b)=(1,0.75)$ and dynamic pressure $\lambda=150$ regarding the order of approximation polynomial series $\left(M_{i}=N_{i}=\widetilde{N}\right)$ and number of in-plane and out-of-plane selected vibration mode shapes for reduced model $\left(n_{\text {in }}=n_{\text {out }}=\tilde{n}\right)$.

\begin{tabular}{lllllr}
\hline & $\tilde{n}=5$ & $\tilde{n}=6$ & $\tilde{n}=7$ & $\tilde{n}=8$ & $\tilde{n}=9$ \\
\hline$\widetilde{N}=5$ & 0.537 & 0.538 & 0.544 & 0.609 & 0.541 \\
$\widetilde{N}=6$ & 0.522 & 0.52 & 0.528 & 0.555 & 0.517 \\
$\widetilde{N}=7$ & 0.522 & 0.522 & 0.528 & 0.555 & 0.52 \\
\hline
\end{tabular}

Table 5: Dimensionless coordinates of points selected on the plate free edge for studying amplitude of limit-cycle oscillations.

\begin{tabular}{lccccc}
\hline & Point 1 & Point 2 & Point 3 & Point 4 & Point 5 \\
\hline$(x / a, y / b)$ & $(1,0)$ & $(1,0.25)$ & $(1,0.5)$ & $(1,0.75)$ & $(1,1)$ \\
\hline
\end{tabular}

\section{Effect of Bonded Patch on Limit-Cycle Oscillations}

According to linear aeroelasticity theory, when dynamic pressure increases monastically, at the beginning plate vibrations are stable, but after passing a critical dynamic pressure, instability arises and vibration amplitude goes to infinity. In practice, existence of nonlinear terms in system governing equations leads the system to a limit-cycle oscillation problem. In order to study effect of embedded patch on limit-cycle oscillations, the piezoelectric patch is bonded on plate leading edge on the free corner, where the maximum critical dynamic pressure is acquired. Since nonlinear analysis can take a huge computation effort, in this section a reduced-order model is employed. For this purpose, firstly nonlinear terms in governing equations are omitted and eigenvectors of the linear method are defined. Then, plate deformation field is approximated using limited numbers of in-plane and out-of-plane mode shapes, as follows [13]:

$$
\left\{\begin{array}{c}
u_{0} \\
v_{0} \\
w_{0}
\end{array}\right\}=r_{1} \mathbf{N} \varphi_{1}+r_{2} \mathbf{N} \varphi_{2}+\cdots+r_{n} \mathbf{N} \varphi_{\mathbf{n}}=\overline{\mathbf{N}} \mathbf{r}
$$

where $r_{1}, r_{2}, \ldots, r_{n}$ are time-dependent coefficients, $\boldsymbol{\varphi}_{1}, \boldsymbol{\varphi}_{2}, \ldots, \boldsymbol{\varphi}_{\mathrm{n}}$ are eigenvectors corresponding to the selected in-plane and out-of-plane mode shapes, $\mathbf{N}$ is matrix of mode shapes corresponding to the original system, $\overline{\mathbf{N}}$ is matrix of mode shapes corresponding to the reduced model, and $\mathbf{r}$ stands for vector of generalized coordinates of the reduced model.

Using the new approximation series presented in the above equation, governing equations of the reduced model are obtained as follows:

$$
\overline{\mathbf{M}} \ddot{\mathbf{r}}+\mathbf{C}_{\mathbf{A}} \dot{\mathbf{r}}+\left(\overline{\mathbf{K}}+\overline{\mathbf{K}}_{\mathrm{q}}+\overline{\mathbf{K}}_{\mathrm{qq}}+\overline{\mathbf{K}}_{\mathrm{A}}\right) \mathbf{r}=\mathbf{0} .
$$

Characteristic matrices introduced by (5.2) are computed using (2.12)-(2.17) by replacing original matrix of mode shapes $\mathbf{N}$ with the one corresponding to the reduced model (i.e., $\overline{\mathbf{N}}$ ). 


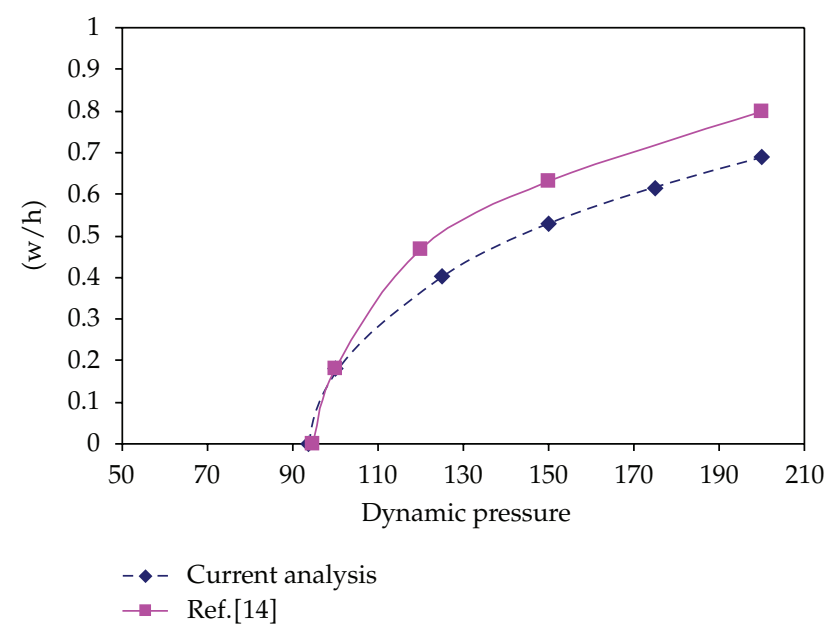

Figure 5: Amplitude of limit-cycle oscillations versus dimensionless dynamic pressure for a cantilevered square plate in dimensionless coordinates $(x / a, y / b)=(1,0.75)$.

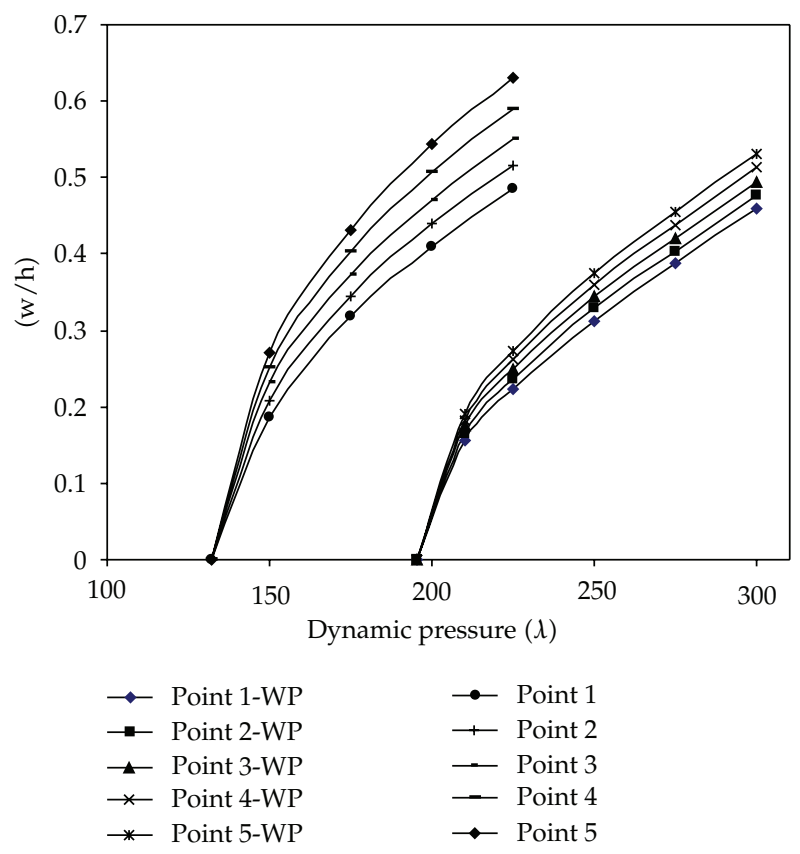

Figure 6: Amplitude of limit-cycle oscillations of a cantilevered plate on the plate free edge for cases with a patch and without a patch in 5 points as reported in Table 4; letters WP represent the case with a bonded patch.

In order to select proper order of approximation series and appropriate number of inplane and out-of-plane vibration modes used for reduced model, a square cantilevered plate is considered. The plate is subjected to a supersonic flow with dynamic pressure $\lambda=150$ and amplitude of limit cycles is measured in dimensionless coordinates $(x, y)=(a, 0.75 b)$. Runge-Kutta direct time integration is used for this analysis. A convergence study on 


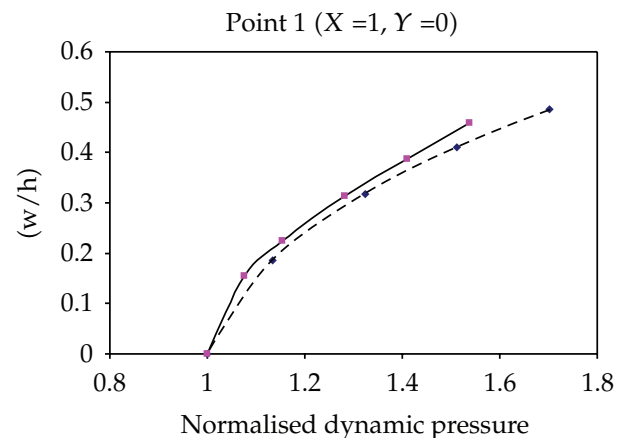

(a)

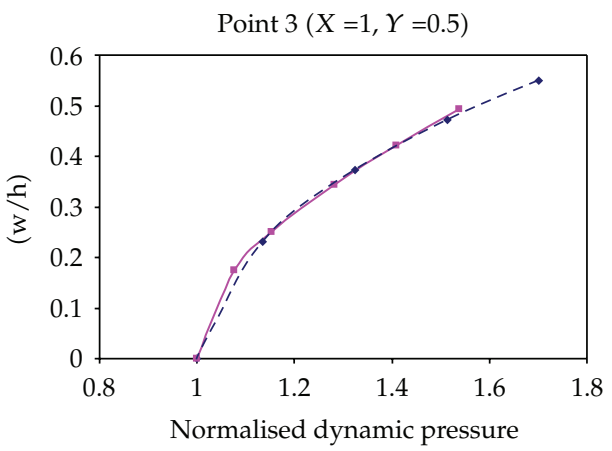

(c)

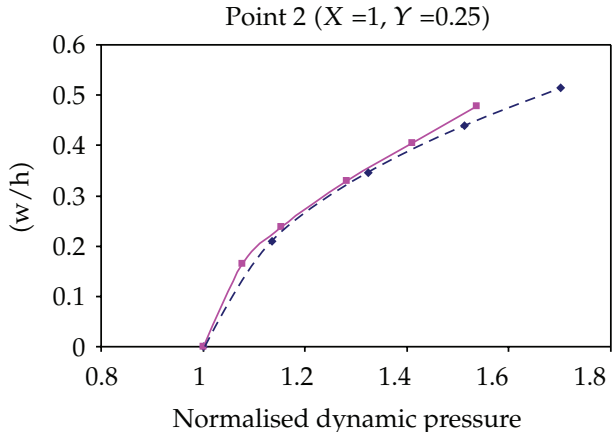

(b)

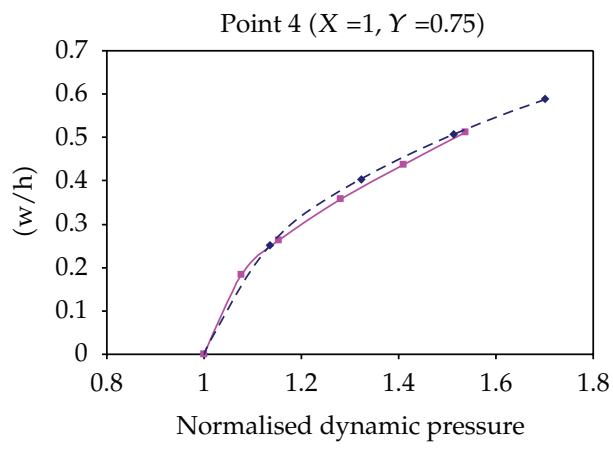

(d)

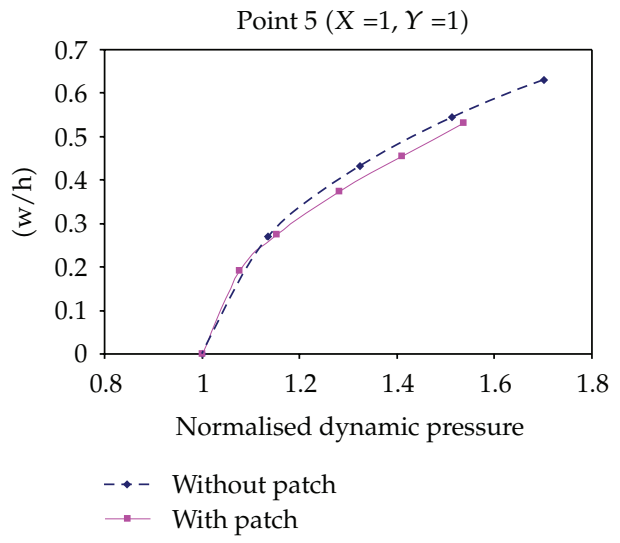

(e)

Figure 7: Amplitude of limit-cycle oscillations of a cantilevered plate on the free edge of the plate versus normalized dimensionless dynamic pressure $\left(\lambda / \lambda_{c r}\right)$. Dimensionless coordinates of the studied points are presented in Table 5.

critical dynamic pressure is performed for a different number of selected modes and order of approximation series, as presented in Table 4. For this convergence study, it has been assumed that orders of polynomial approximation series in $x$ and $y$ directions are the same $\left(M_{i}=N_{i}=\widetilde{N}, i=1,2,3\right)$. In addition, it has been assumed that a number of selected in-plane and out-of-plane mode shapes are equal $\left(n_{\text {in }}=n_{\text {out }}=\tilde{n}\right)$. From this table, it is observed that 
using polynomial series of order $\widetilde{N}=7$ and selected mode numbers $\widetilde{n}=7$ results in a good convergence in obtained solution. Additionally, using larger number of applied mode shapes may disturb the convergence (e.g., $\tilde{n}=8$ ).

Figure 5 presents a comparison between limit-cycle amplitudes found by the current analysis with those of [14]. This comparison illustrates some differences between the obtained results. Since number of mode shapes employed in [14] is rather small and in-plane inertia forces are ignored there, some differences in the results are justifiable.

With the aim of studying effect of patch placement on limit-cycle oscillations and vibration mode shapes, a cantilevered plate with dimensions presented in (4.2) is considered. Two cases are considered for this plate, namely, without patch and with a patch having a size introduced by (4.3). Five points on the free edge of the plate are selected. Table 5 presents dimensionless coordinates of these points. Amplitude of limit-cycle oscillations corresponding to the reported points is illustrated in Figure 6, where letters WP indicate the case of cantilevered plate with an embedded patch. This figure clearly shows that bonding a patch on the plate free corner in the leading edge delays critical dynamic pressure and raise of limit-cycle amplitudes.

In order to have a better understanding of mode shape variations, limit-cycle amplitudes are illustrated versus normalized dynamic pressure in Figure 7. This figure shows that, by bonding a patch in the mentioned coordinates and in comparison with the case without a patch, amplitude of limit cycles reduces moving from leading edge to trailing edge (i.e., from point 1 to point 5). This indicates a slight change in vibration mode shape. It is clear that variation of mode shape results in a change in aerodynamic couplings. Therefore, variation of aeroelastic behavior of plate is expectable.

\section{Conclusion}

In this paper, effect of patch placement on aeroelastic behavior of cantilevered plates is studied. Results show that, for this type of plates, bonding a patch with a small mass ratio changes critical dynamic pressure considerably. This yields a significant change in the dynamic of the system. A survey of the literature shows that this effect is ignored by lots of researchers. Evidently, such an important change in the system behavior demands some advanced methods for optimal actuator placement which respect system controllability minimization beside system passive performance improvement. Alternatively, investigators can explore patch location for optimal passive and active flutter controls, in parallel, and assess the most suitable one or combine the obtained results to develop a hybrid control methodology.

\section{References}

[1] S. H. Moon and S. J. Kim, "Active and passive suppressions of nonlinear panel flutter using finite element method," AIAA Journal, vol. 39, no. 11, pp. 2042-2050, 2001.

[2] K. Abdel-Motagaly, X. Guo, B. Duan, and C. Mei, "Active control of nonlinear panel flutter under yawed supersonic flow," AIAA Journal, vol. 43, no. 3, pp. 671-680, 2005.

[3] S. H. Moon, "Finite element analysis and design of control system with feedback output using piezoelectric sensor/actuator for panel flutter suppression," Finite Elements in Analysis and Design, vol. 42, no. 12, pp. 1071-1078, 2006.

[4] J.-H. Han, J. Tani, and J. Qiu, "Active flutter suppression of a lifting surface using piezoelectric actuation and modern control theory," Journal of Sound and Vibration, vol. 291, no. 3-5, pp. 706-722, 2006. 
[5] C. Nam, Y. Kim, and T. A. Weisshaar, "Optimal sizing and placement of piezo-actuators for active flutter suppression," Smart Materials and Structures, vol. 5, no. 2, pp. 216-224, 1996.

[6] R. E. Richard and R. L. Clark, "Delta wing flutter control using spatially optimized transducers," Journal of Intelligent Material Systems and Structures, vol. 14, no. 11, pp. 677-691, 2003.

[7] T. Von Karman, "Festigkeitsproblems im Maschinenbau," Encyclopadie der mathematischen Wissenschafter, vol. 4, pp. 384-352, 1910.

[8] M. Amabili and S. Farhadi, "Shear deformable versus classical theories for nonlinear vibrations of rectangular isotropic and laminated composite plates," Journal of Sound and Vibration, vol. 320, no. 3, pp. 649-667, 2009.

[9] T. Kane and D. Levinson, Dynamics: Theory and Application, McGraw-Hill, New York, NY, USA, 1985.

[10] G. Sander, C. Bon, and M. Geradin, "Finite element analysis of supersonic panel flutter," International Journal for Numerical Methods in Engineering, vol. 7, no. 3, pp. 379-394, 1973.

[11] C. Mei, K. Abdel-Motagaly, and R. Chen, "Review of nonlinear panel flutter at supersonic and hypersonic speeds," Applied Mechanics Reviews, vol. 52, no. 10, pp. 416-421, 1999.

[12] N. Mallik and M. C. Ray, "Effective coefficients of piezoelectric fiber-reinforced composites," AIAA Journal, vol. 41, no. 4, pp. 704-710, 2003.

[13] M. Amabili, Nonlinear Vibrations and Stability of Shells and Plates, Cambridge University Press, New York, NY, USA, 2008.

[14] Y. Weiliang and E. Dowell, "Limit cycle oscillation of a fluttering cantilever plate," AIAA Journal, vol. 29, no. 11, pp. 1929-1936, 1991. 


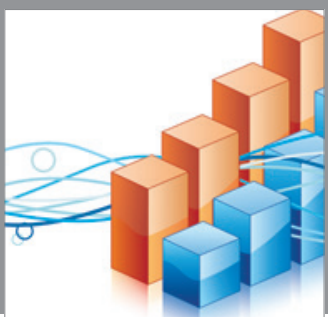

Advances in

Operations Research

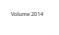

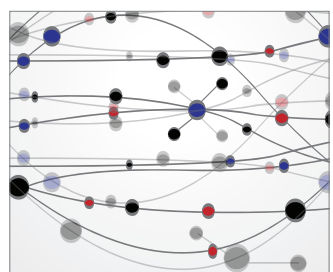

\section{The Scientific} World Journal
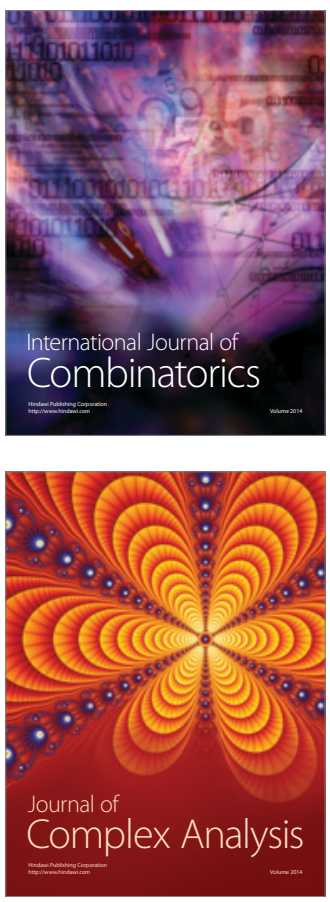

International Journal of

Mathematics and

Mathematical

Sciences
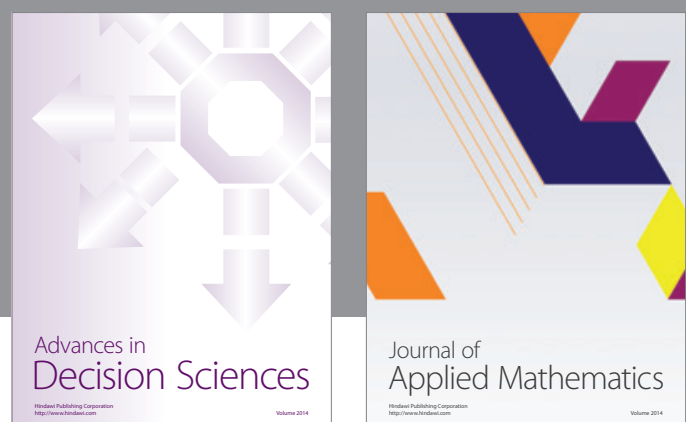

Journal of

Applied Mathematics
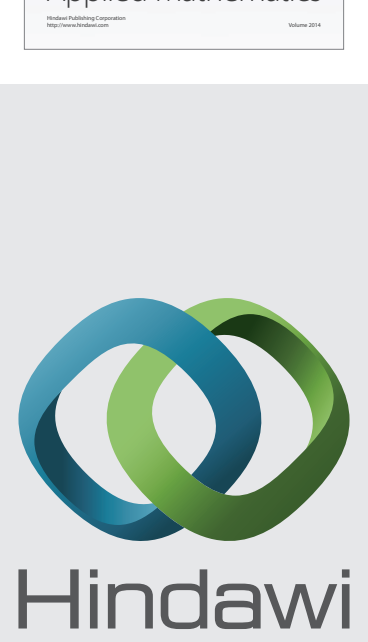

Submit your manuscripts at http://www.hindawi.com
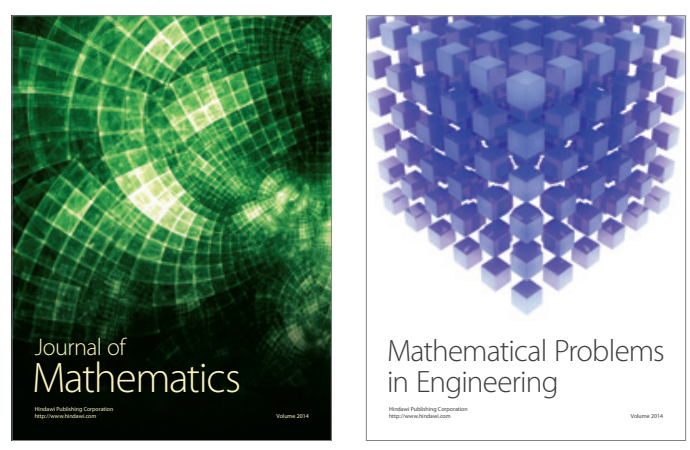

Mathematical Problems in Engineering
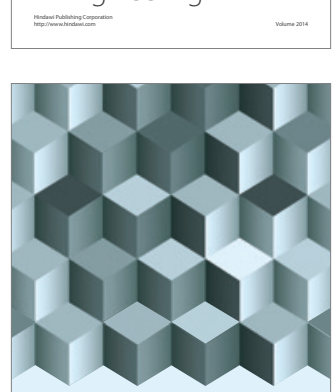

Journal of

Function Spaces
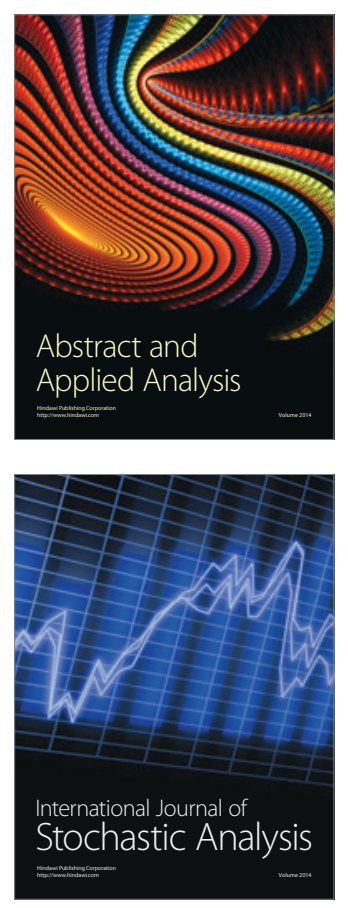

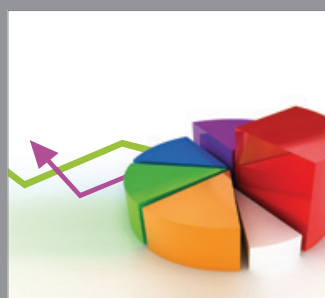

ournal of

Probability and Statistics

Promensencen
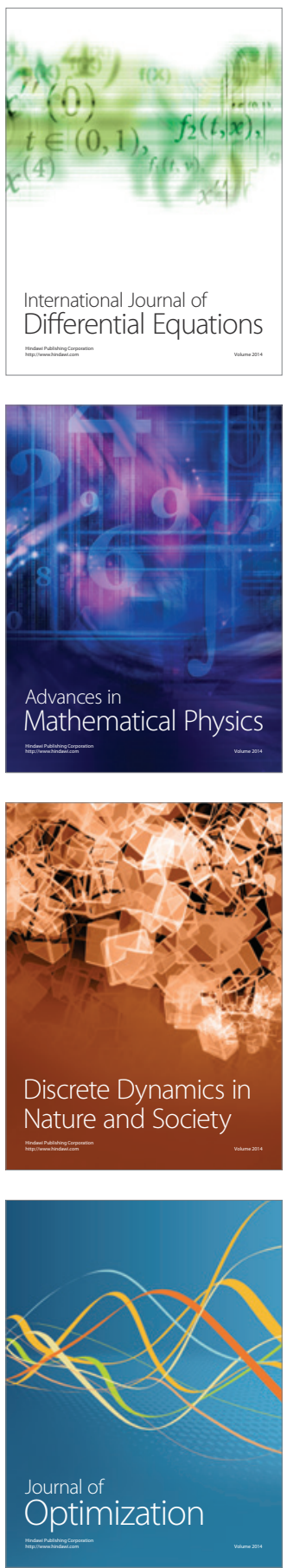